\title{
Anisotropic thermophoresis
}

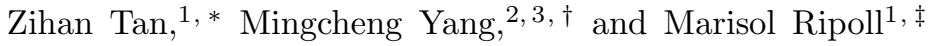 \\ ${ }^{1}$ Theoretical Soft-Matter and Biophysics, Institute of Complex Systems, \\ Forschungszentrum Jülich, 52425 Jülich, Germany \\ ${ }^{2}$ Beijing National Laboratory for Condensed Matter Physics and Key Laboratory of Soft Matter Physics, \\ Institute of Physics, Chinese Academy of Sciences, Beijing 100190, China \\ ${ }^{3}$ University of Chinese Academy of Sciences, Beijing 100049, China
}

(Dated: October 14, 2018)

\begin{abstract}
Colloidal migration in temperature gradient is referred to as thermophoresis. In contrast to particles with spherical shape, we show that elongated colloids may have a thermophoretic response that varies with the colloid orientation. Remarkably, this can translate into a non-vanishing thermophoretic force in the direction perpendicular to the temperature gradient. Oppositely to the friction force, the thermophoretic force of a rod oriented with the temperature gradient can be larger or smaller than when oriented perpendicular to it. The precise anisotropic thermophoretic behavior clearly depends on the colloidal rod aspect ratio, and also on its surface details, which provides an interesting tunability to the devices constructed based on this principle. By means of mesoscale hydrodynamic simulations, we characterize this effect for different types of rod-like colloids.
\end{abstract}

\section{INTRODUCTION}

The migration of particles in the presence of a temperature gradient was first described in the XIX century [1, 2] and it is generally known as thermal diffusion or Soret effect. The study of the Soret effect in liquid mixtures is usually referred to as thermodiffusion [3. In these mixtures, all relevant particles sizes are similar and within the nanometer scale. The same physical principle applied to colloids is typically referred to as thermophoresis [4, 5]. Colloids can have sizes up to the micrometer scale and the surrounding solvent particles can be orders of magnitude smaller. Several factors are known to importantly influence the thermodiffusive behavior of these systems like the colloid size, mass, average temperature, concentration [3, 4. In fluid mixtures the effect of the particle moment of inertia has been extensively studied [6] 8 , concluding that increased moment of inertia facilitates migration. Rodlike colloids have been experimentally investigated [9, 10] and characterized as a function of their electrostatic interactions. However, no systematic study has been yet done to investigate the thermophoretic properties of colloids as a function of their shape.

Most colloids show thermophobic behavior (average migration to the cold areas), although thermophilic colloids have also been frequently reported [11, 12]. In both cases, the colloid migration is characterized by a unique value of the so-called thermal diffusion factor which, by convection, is positive for thermophobic colloids and negative for thermophilic. The sign and the value of the thermodiffusion factor is determined by the colloid size and properties of the colloid solvent interactions, and it can be modified or even reversed for example by changing

\footnotetext{
* z.tan@fz-juelich.de

$\dagger$ mcyang@iphy.ac.cn

$\ddagger$ m.ripoll@fz-juelich.de
}

average temperature, concentration, $\mathrm{pH}$ or solvent composition. But the question that arises is how is the thermophoretic behavior of elongated particles. A valuable and interesting parallelism can be made here between thermophoretic and friction forces. An elongated object moving in a fluid along its axis is known to experience frictional force $\gamma_{\|}$, which is typically much smaller than the friction experienced by the same rod moving perpendicular to its axis $\gamma_{\perp}$. This is a well-know fact, which in the case of a shish-kebab model of adjacent beads has been calculated to be $\gamma_{\perp}=2 \gamma_{\|}$for aspect ratios larger than 20 [13. It is therefore intuitive, that an elongated object with its axis aligned with a temperature gradient, will not have the same thermophoretic response as when the axis is perpendicular to the gradient. Hence and in contrast to colloids with spherical symmetry, colloids with an anisotropic shape should be characterized by two or more thermal diffusion factors, which is the main concept of the anisotropic thermophoresis [14].

Practical applications of thermodiffusion have developed over several decades and are currently in a significant expansion stage. Some relevant examples are crude oil characterization [15, separation techniques [16], strong components accumulation in prebiotic conditions [17, 18, the precise characterization of proteins, for which thermophoresis can even distinguish betweeen different binding states [19]; also various application in microfluidics [20, 21, or the fabrication of synthetic microswimmers 22,24 . The traditional versatility of thermophoresis is therefore importantly increased by considering different shaped objects.

In this work we investigate the anisotropic thermophoretic properties of colloidal rods by means of hydrodynamic computer simulations. We study the dependence of the thermophoretic forces for moving rods and for fixed rods at different orientations, with various aspect ratios and surface properties. The anisotropic effect can for example be reversed by changing the surface ru- 
gosity, which can be understood in terms of the associated temperature properties of the fluid in the vicinity of the colloid. Interestingly, this anisotropy can induce a thermophoretic effect non-aligned with the temperature gradient. This contribution to the thermophoretic force perpendicular to the temperature gradient has already shown to be the basic mechanism that allows the construction of thermophoretic turbines, which move in the presence of an external temperature gradient [14]. Other promising applications are not yet explored, but are certainly to be expected, and are specially promising in microfluidic devices where significantly large and welllocalized temperature gradients can be generated and precisely controlled in time and space. Applications of anisotropic thermophoresis in the presence of external temperature gradients offer then the possibility of engineering devices able to harvest waste heat energy.

\section{SIMULATION METHOD}

In order to be able to bridge the time and length scales of the colloidal rods and the surrounding solvent particles, a hybrid description of the suspension is employed. The solvent is simulated by an efficient technique known as multiparticle-collision dynamics (MPC) 25] 27, while the colloidal rod is described with a Molecular Dynamics simulation model. In MPC, the solvent is represented by $N$ point particles whose dynamics takes place in two sequential steps. One is the streaming step, at which all point particles move ballistically for a certain collision time $h$. In the collision step, particles are grouped into cubic collision cells of size $a$, where particles interchange momentum by performing a rotation by a certain angle $\alpha$ of the particle velocity relative to the corresponding cell center of mass velocity. MPC accounts for thermal fluctuations, and conserves energy and linear momentum both globally and locally [28 30] by construction. Angular momentum is not conserved for this MPC implementation [31, 32. However, recent work has provided evidence that angular momentum conservation in MPC fluid does not influence the hydrodynamics of phoretic flows 33. Simulations are performed with an average of $\rho=10$ particles per collision cell, the collision time step $h=0.1$, the rotation angle $\alpha=130^{\circ}$, and the average temperature of the solvent $k_{B} \bar{T}=1$. These parameters yield the viscosity $\eta=8.7$ according to the theoretical predictions [26, 28, 34 38, which is in close agreement with simulation results 39. In the following, all quantities are expressed in terms of the MPC units, where the units of length, mass and energy are separately imposed as $a, m$ and $k_{B} \bar{T}$, such that the time unit is $a \sqrt{m / k_{B} \bar{T}}$. The temperature gradient is applied in the $z$ direction with a boundary thermostat [40 42], ensuring that heat conduction is correctly accounted for.

The rod is constructed with the "shish-kebab" model built by $N$ connected beads in a linear disposition as shown in Fig. 1. The excluded volume interactions between colloid and solvent are performed via MD with Lennard-Jones (LJ) type potentials [43, 44]

$$
U_{k}(r)=4 \epsilon\left[\left(\frac{d}{2 r}\right)^{2 n}-\left(\frac{d}{2 r}\right)^{n}+c\right], r \leq r_{c} .
$$

Here $r$ is the distance between the bead center and the fluid particle, $\epsilon$ refers to the potential intensity, $d$ to the bead diameter, and $n$ is a positive integer describing the potential stiffness. The repulsive or attractive LJ potentials are obtained by considering $c=1 / 4$ or $c=0$ respectively, together with the adequate cutoff distance $r_{c}$. The repulsive and attractive potentials with stiffness $n=6$ will be denoted as $r 6$ and $a 6$ respectively, and similar for other $n$ values. The bead diameter is taken as

$d=4 a$, and $\epsilon=k_{B} \bar{T}$. The mass of each bead is chosen such that the rod is neutrally buoyant, although results are not really depending on this value. The equations of motion of the beads and interacting fluid are integrated with a velocity-Verlet MD algorithm. The rod length

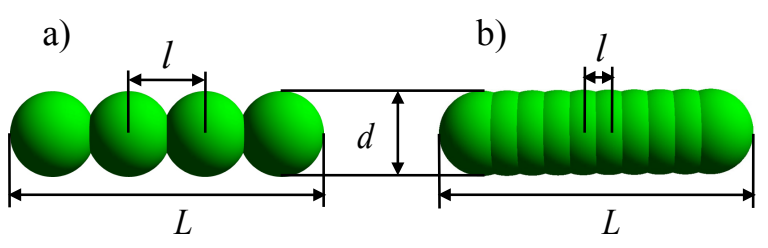

FIG. 1. Sketch of the "shish-kebab" model of a colloidal rod of aspect ratio $L / d=3.7$ with variable number of beads $N$, and variable inter-bead separation $l$. (a) "Rough" rod with $N=4$ beads and $l=0.9 d$. (b) "Smooth" $\operatorname{rod}$ with $N=10$ beads and $l=0.3 d$.

$L$ is given by $L=d+(N-1) l$, such that the aspect ratio $L / d$ can be fixed for more than one combination of $N$ and $l$. Besides the particular solvent-bead potential, the rod surface properties are going to be determined by the interbead separtion, which we characterize with $l / d$, the rugosity parameter. As can be seen in Fig. 1. a rough rod is obtained when $l \simeq d$, while a smooth rod is obtained when $l \ll d$. In order to prevent the penetration of the fluid inside the rod, the maximum value of the rugosity parameter we employ is $l / d=0.9$. We consider a three-dimensional box with periodic boundary conditions changing from 3 to 4 times the rod length $L$. Two types of simulations are performed. Simulations with fixed rods only need the collod solvent interactions to be specified. Simulation with freely rotating rods and fixed central of mass, in which the rod motion is additionally accounted with rigid body dynamics 45 .

\section{RESULTS}

The migration of a particle in a temperature gradient is driven by the thermophoretic force $\mathbf{F}_{T}$ [46] 
force arises from the interactions between the particle and the surrounding inhomogeneous fluid environment. In the case of spherically symmetric colloids, this force is characterized by a unique value of the so-called thermodiffusion factor, $\alpha_{T}$, as

$$
\mathbf{F}_{T}=-\alpha_{T} k_{B} \nabla T
$$

with $k_{B}$ the Boltzmann constant, and $\nabla T$ the temperature gradient. There is though no reason to expect that a unique factor would still describe the thermophoretic behavior of an non-spherically symmetric particle. A straightforward generalization can be written in terms of the thermodiffusion tensor $\Lambda_{T}$ as

$$
\mathbf{F}_{T}=-\Lambda_{T} \cdot \nabla T .
$$

In a very general case, colloids with arbitrary shape and arbitrary surface properties can be defined by a symmetric tensor with independent coefficients. Of particular interest is the case of an homogeneous colloidal rod with cylindrical symmetry. In this case, two independent coefficients are expected to be enough to determine the thermodiffusion tensor as

$$
\Lambda_{T}=\alpha_{T, \|} \hat{\mathbf{u}} \hat{\mathbf{u}}+\alpha_{T, \perp}(\hat{\mathbf{I}}-\hat{\mathbf{u}} \hat{\mathbf{u}}),
$$

with $\hat{\mathbf{u}}$ the unit vector of long axis of $\operatorname{rod}$. Here $\alpha_{T, \|}$ is the thermodiffusion factor of the long rod axis, or equivalently the thermophoretic factor that characterizes a rod with the long axis aligned with the temperature gradient, as displayed in Fig. 2 2 . Reciprocally, $\alpha_{T, \perp}$ is the thermodiffusion factor of the short rod axis, or of a rod with the long axis oriented perpendicular to the temperature gradient (see Fig. 2b).

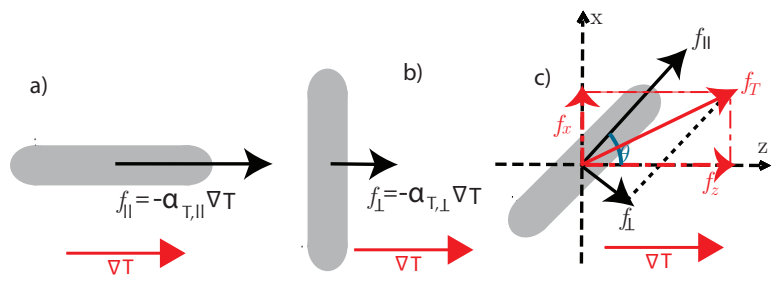

FIG. 2. Sketch of the thermophoretic force of a colloidal rod fixed with different orientations with respect to the temperature gradient: a) parallel, b) perpendicular, c) with an arbitrary $\theta$ angle.

a. Linear decomposition of the thermophoretic force.The thermophoretic force acting on a rod with arbitrary orientation can be determined by the linear superposition of the two components with orthogonal thermodiffusion factors $\mathbf{F}_{T}=\mathbf{F}_{\|}+\mathbf{F}_{\|}$. The temperature gradients along the long and short axis are respectively $\nabla T_{l}=\cos \theta \nabla T$ and $\nabla T_{s}=\sin \theta \nabla T$, with $\theta$ the angle between the particle long axis and the temperature gradient, as displayed in Fig. 2k. The total force can then be expressed in terms of its components, parallel and perpendicular to the temperature gradient $\mathbf{F}_{T}=\mathbf{F}_{z}+\mathbf{F}_{x}$ as,

$$
\begin{aligned}
& \mathbf{F}_{z}=-\left(\alpha_{T, \perp} \sin ^{2} \theta+\alpha_{T, \|} \cos ^{2} \theta\right) k_{B} \nabla T, \\
& \mathbf{F}_{x}=\left(\alpha_{T, \perp}-\alpha_{T, \|}\right) \sin \theta \cos \theta k_{B}|\nabla T| \mathbf{n}_{x}
\end{aligned}
$$

where $\mathbf{n}_{x}$ is the unit vector perpendicular to $\nabla T$. Equation (6) comes as a straightforward result of the tensorial character of the thermodiffusion tensor in Eq. (3), and strikingly implies that a non-vanishing thermophoretic force exists in the direction perpendicular to the temperature gradient. This force can in fact be easily measured in our simulations as shown in Fig. 3, such that it could also be measured experimentally [49, 50]. The measured force perpendicular to the temperature gradient is cleary non-vanishing, and it increases linearly with the applied tempeature gradient, as expected from Eq. (6). This nicely confirms the tensorial character of the thermophoretic effect for objects without spherical symmetry.

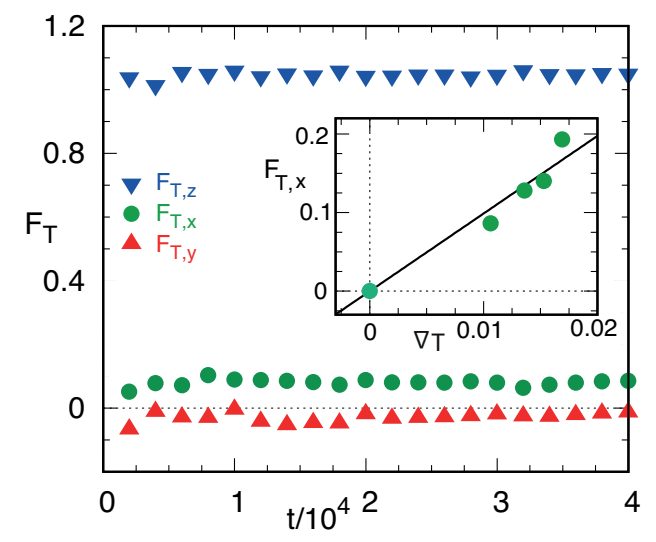

FIG. 3. Thermophoretic force obtained from simulations as a time average for a fixed smooth $\operatorname{rod}(l / d=0.3)$, of aspect ratio $L / d=3.7$, and interaction potential $r 6$. The rod is fixed with an angle $\theta=45^{\circ}$ with respect to $\nabla T$. The force perpendicular to $\nabla T, F_{T, x}$, is non-vanishing as predicted by Eq. (6). The inset shows the value of $F_{T, x}$ for various values of $\nabla T$.

Simulations with single rods fixed by an angle $\theta$ with respect to the temperature gradient are performed for different orientations, as shown in Fig. 4. The values of $\alpha_{T, \|}$ and $\alpha_{T, \perp}$ can be obtained by fitting the expressions Eqs. (5) and (6) to the simulation results, or more efficiently, just by fixing the rod parallel $(\theta=0)$ or perpendicular $(\theta=\pi / 2)$ to the temperature gradient. The linear decomposition of the thermophoretic force in Eqs. (5) and (6) is clearly verified by these simulation results.

Results in Fig. 4 also show that, depending on the simulated rods, $F_{T, \|}$ can be larger or smaller than $F_{T, \perp}$, such that the force perpendicular to the gradient, $F_{T, x}$, can appear in both directions. This is in strong contrast to the friction force, which is always larger for rods 


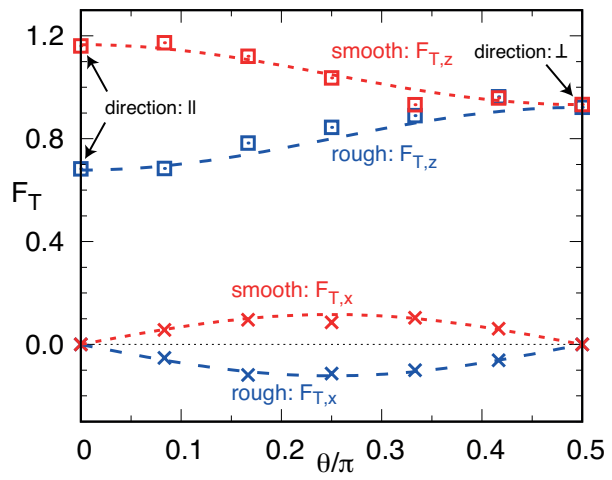

FIG. 4. Thermophoretic force for fixed smooth $(l / d=0.3)$ and rough $(l / d=0.9)$ rods with $L / d=3.7$ and $r 6$. The angle $\theta$ denotes the rod orientation with respect to $\nabla T$, as sketched in Fig. 22. The force $F_{T, z}$ is measured in the direction paralle to $\nabla T$ and $F_{T, x}$ perpendicular to it. Symbols correspond to simulation results and lines to the expressions in Eq. (5) and 6.

oriented perpendicular to the flow than for those parallel to it. Hence, the anisotropic thermophoretic effect is fundamentally different from the anisotropy of the translational friction.

b. Does thermophoretic anisotropy induce orientation? As just discussed, elongated particles fixed aligned with the temperature gradient or perpendicular to it, can experience well-differentiated thermophoretic forces. Nevertheless, a freely moving rod in a temperature gradient, suffers a force exerted on one half of the rod which is exactly the same as in the other half, such that there is no net torque on the particle. For the same reason, friction forces are also known not to induce any orientation effects on elongated particles, in spite of their anisotropy. Orientation is induced only in the case that the flow field is in itself not homogeneous, as it is the case of a shear flow. The thermophoretic anisotropy does therefore induce no particle alignment. A freely rotating rod in a temperature gradient, will then change its orientation only due to stochastic interactions. Simulations allowing particle rotation confirm this statement for the two parameter sets in Fig. 4, and the measured thermodiffusion factors verify that $\alpha_{T, \text { free }}=\left.\left\langle\alpha_{T}\right\rangle_{\theta} \simeq \alpha_{T, \|}\right|_{\theta=45}$.

While alignment refers to the first moment of the induced orientation, we could wonder if the effect exist in higher moment orders. It is therefore interesting to investigate if the presence of a external temperature gradient could modify the particle rotational diffusion. We measure the rotational diffusion coefficient by characterizing the long time behaviour of the mean squared orientation displacement. For computational efficiency, these simulations consider rods composed of smaller beads, $d=2 a$. The normalizing factor $D_{r}^{0} \simeq 8 \times 10^{-4}$ is obtained for the rough rods in the absence of temperature gradient. In the presence of non-vanishing temperature gradients, simulations are performed by keeping the rod center of mass fixed in the middle of the simulation box where the solvent average temperature is $k_{B} \bar{T}=1$. The simulation

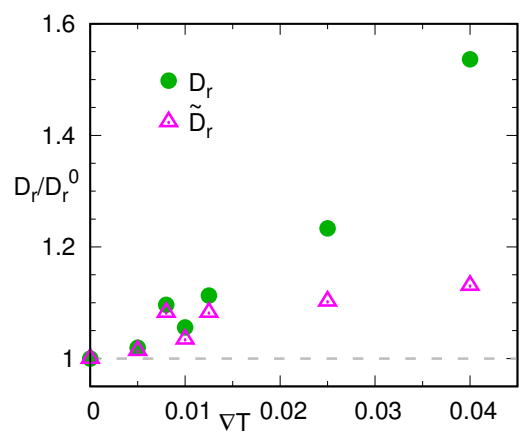

FIG. 5. Normalized diffusion coefficient for a freely rotating $\operatorname{rod}($ with $l / d=0.3, L / d=3.7$, and $r 6$ ) at various temperature gradients. Bullets correspond to the direct simulation measurements $D_{r}$, and triangles to the coefficient rescaled with the density correction $\tilde{D}_{r}$.

data in Fig. 5 show to increase with the applied temperature, but this is mainly due to the ideal gas character of the employed MPC fluid. Given a spatial linear increase of the temperature, the related position dependent density will be only approximately linear 40. The density in the middle of the simulation box will then differ from the average one, which will affect the rotational diffusion coefficient. If we consider that $D_{r} \propto 1 / \eta$, and that $\eta \propto(\rho-1)$ [51] we can explain the major $D_{r}$ by considering the rescaled coefficient $\tilde{D}_{r}=D_{r}\left(\left.\rho\right|_{L_{Z} / 2}-1\right) /(\rho-1)$. The factor $\left.\rho\right|_{L_{Z} / 2}$ can be measured in the simulations, or calculated from the ideal gas equation of state as $\rho /\left.\rho\right|_{L_{Z} / 2}=\bar{T} \ln \left(T_{h} / T_{c}\right) /\left(T_{h}-T_{c}\right)$ [40]. The rescaled coefficient $\tilde{D}_{r}$ in Fig. 5 shows to be mostly independent of the applied temperature gradient within the precision of the data, which demonstrates that the temperature gradient has no effect in the particle orientation.

It is interesting to mention that thermomolecular orientation has been previously reported in diatomic fluids 52 54. In these cases, elongated molecules made of two atoms with unequal sizes show to display certain average orientation towards the direction of the temperature gradient. This does not contradict the discussed lack of orientation induced by anisotropic thermophoresis, since the particules we discuss are elongated but intrinsically symmetric, namely composed of indistinguisable building blocks.

c. Aspect ratio effect.- For spherical colloids, the size is well-known to influence $\alpha_{T}$, the particle thermodiffusion factor [44, 55, 56. The overall increase of size of the rod will therefore also translate into an increase of $\alpha_{T}$, but how does the rod aspect ratio influence the anisotropic effect, still needs to be clarified. Simulations of rods with orientations parallel and perpendicular to the temperature gradient are performed for different aspect ratios and for two rugosities as shown in Fig. 6. Obtained measurements are normalized with $\alpha_{T}^{0}$, the thermal diffusion factor of a single bead of the same char- 


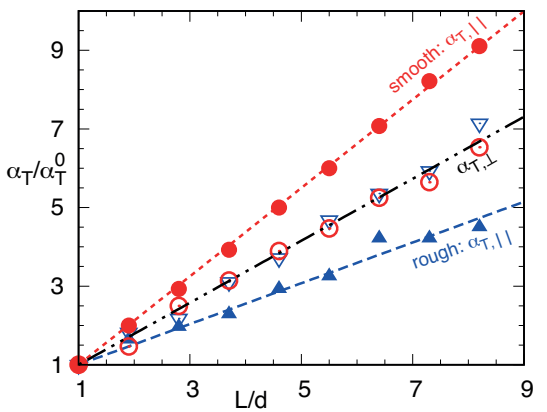

FIG. 6. Normalized parallel (open symbols) and perpendicular (filled symbols) thermodiffusion factors as a function of the aspect ratio $L / d$ for smooth and rough rods (rugosity parameters $l / d=0.3$ and 0.9$)$ with $r 6$ interaction potential.

acteristics as the ones employed to build the rod, such that for $L=d, \alpha_{T, \perp}=\alpha_{T, \|}=\alpha_{T}^{0}$ by definition. The four analyzed cases in Fig. 6 show a very clear linear increase of $\alpha_{T}$ with the aspect ratio $L / d$, although with different slopes. The linear increase can be easily understood since the increase of the rod surface exposed to the temperature gradient also increases linearly. This means that by characterizing the values of $\alpha_{T}$ at two different aspect ratios with enough precision, would allow us to easily extrapolate to other aspect ratios; also in the case that one of those values is the limiting spherical case $\alpha_{T}^{0}$. In the case of a freely rotating colloidal rod, the overall thermodiffusion factor will therefore also increase linearly with the aspect ratio of the colloidal rod. This is very interesting by itself, and reminiscent of the well-known effect of the particle moment of inertia on the thermal diffusion in molecular mixtures [6] 8 .

d. Surface effects.- The rod surface is modified in our model in two different manners. On the one hand, the surface shape as described in Fig. 11 modifies its rugosity with the parameter $l / d$. On the other hand, the choice of the employed potential, attractive-repulsive, soft-steep will also modify the thermophoretic properties of the rod. To analyze more in detail these effects, further simulations calculate $\alpha_{T, \|}$ and $\alpha_{T, \perp}$ as a function of the different potential interactions and the rugosity parameter $l / d$, as shown in Fig. 7 and Fig. 8. In the case of spherical colloids, the thermodiffusion factor $\alpha_{T}^{0}$ is known to depend on the colloid-surface interactions, and on the colloid size 4, 44. With rods of fixed aspect ratio, we perform simulations with repulsive and attractive potentials, and with different $n$ values in Eq. 11, for which $\alpha_{T}^{0}$ is also evaluated. The parallel $\alpha_{T, \|}$, and perpendicular $\alpha_{T, \perp}$, thermodiffusion factors are compared with $\alpha_{T}^{0}$, as displayed in Fig. 7. Values larger than the reference linear increase in Fig. 7, in absolute numbers, indicate the enhancement of the thermal diffusion factors due to the increase elongation of the rod. This enhancement is clear in all investigated cases, although its magnitude differs for the different potentials, rugosities, and orientations. In general, the increase of $\alpha_{T, \|}$ and $\alpha_{T, \perp}$ shows to be

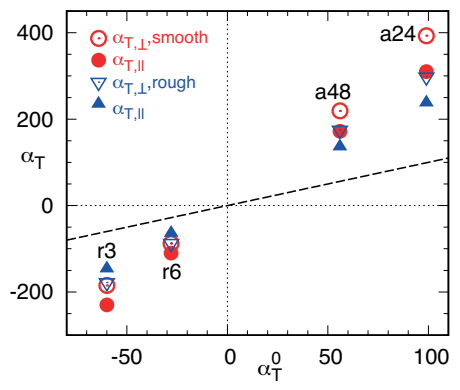

FIG. 7. Parallel and perpendicular thermodiffusion factors for rods with $L / d=3.7$ with various interaction potentials (indicated in the labels) as a function of $\alpha_{T}^{0}$, the thermodiffusion factor of a single bead. Results are provided for the same two rugosity parameters as in Fig. 6. The dashed line is a guide to the eye.

larger, the larger the $\alpha_{T}^{0}$.

The effect of the surface rugosity can already be observed in Fig. 4 and Fig. 6 where simulation results of two well-differentiated rod rugosities are presented. For the repulsive potential and aspect ratio here employed, the rough rod shows $\left|\alpha_{T, \|}\right|<\left|\alpha_{T, \perp}\right| ; i$. e. the thermophoretic force for the rod aligned with the temperature gradient is smaller than for the perpendicular one. Meanwhile the smooth rod with the same potential shows the opposite behavior, $\left|\alpha_{T, \|}\right|>\left|\alpha_{T, \perp}\right|$. This means that by changing the rugosity of the rod, the forces perpendicular to the temperature gradient can invert their direction, as shown in Fig. 4. This is though not the case for the rods simulated with attractive interactions where $\alpha_{T, \|}<\alpha_{T, \perp}$ for both the smooth and the rough surfaces. Note that the sign of the thermodiffusive factors is never modified, such that the thermophilic/thermophobic character of the colloids does not change with its shape change from spherical into elongated, and it will be therfore the same for both components $\alpha_{T, \|}$ and $\alpha_{T, \perp}$.

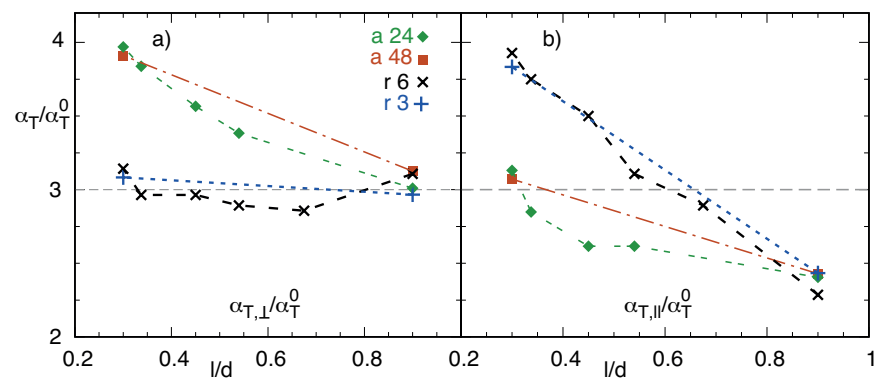

FIG. 8. Normalized parallel and perpendicular thermodiffusion factors for rods with $L / d=3.7$ as a function of the rugosity parameter $l / d$. Simulations with various interaction potentials are reported and indicated in the corresponding labels. The horizontal line is a guide to the eye.

The dependence of the thermal diffusion factors with the rugosity parameter $l / d$, is shown in Fig. 8. With increasing rugosity of the rod surface, the thermal diffu- 
sion factor shows to decrease, or to remain unchanged. In other words, smoother surfaces produce larger thermophoretic responses or eventually similar than their rough counterparts. The clear decrease of $\alpha_{T, \|}$ with increasing rugosity is what eventually changes the relative importance of both factors and the direction of the force perpendicular to the temperature gradient.

To explain these dependencies, we should discuss two types of contributions, which are again related to the rod-solvent interaction potentials and the surface shape. The finite range of the solvent-bead interactions results in overlapping regions, in which solvent particles can simultaneously interact with more than one neighboring bead. The size of these overlapping areas decreases with increasing roughness, or potential steepness, modifying the effective rod-solvent potential. This effect is smaller for rough rods, for which the thermodiffusion factors in Fig. 8 have little dependence of the type of potential. Smooth rods interestingly show a difference between attractive (thermophobic) potentials, and repulsive (thermophilic) ones, but not between those with different steepness. The second type of contribution is in this case dominant, and related to the surface shape, and in particular with the presence of surface indentations which can explain the decrease of $\alpha_{T, \|}$ with increasing roughness. To estimate the contribution to thermophoretic force between two arbitrary points at the rod surface, we consider first that the thermophoretic factor along a wall, can be assumed to be directly proportional to the wall length, which has been shown in Fig. 6. By considering Eq. (2), and that the gradient depends on the inverse of the distance, the contribution to thermophoretic force can then be determined by the difference of temperatures between these two points $T_{c}$ and $T_{h}$. Figure 9 illustrates the thermophoretic forces along a wall with an indentation, $F_{A}$, and a flat wall $F_{B}$, which are both considered to be the sum of two contributions by considering the temperature at the middle of the wall $T_{m}$. Indented and flat walls have different lengths, but the temperatures at their ends are the same. The wall length increase exactly cancels with the decrease of the temperature gradient, such that the wall thermophoretic forces are precesely the same, this is $F_{A 1}=F_{B 1}$ and $F_{A 2}=F_{B 2}$. The total force in both cases is though not the same due to the angle $\theta$ that determines the indentation, as sketched in Fig. 9, such that the force in the indented surface $F_{A}$ is a factor $\cos \theta$ smaller than the force in the perfectly smooth surface $F_{B}=F_{A} / \cos \theta$. This effect is very clear when the rod is aligned with the temperature gradient, and explains the clear decrease of $\alpha_{T, \|}$ with $l / d$ for all potentials in Fig. $8 \mathrm{p}$. When the rod is perpendicular to the gradient, the effect of the indentation is subtly different but still present due to employed three-dimensional structure. However, the overall contribution is smaller which explains the unchanged or decreasing dependence of $\alpha_{T, \perp}$ with $l / d$ in Fig. 8 a .

e. Thermophoretic anisotropy factor.- The importance of the anisotropic effect is determined by how different are the thermophoretic forces of the rods aligned and

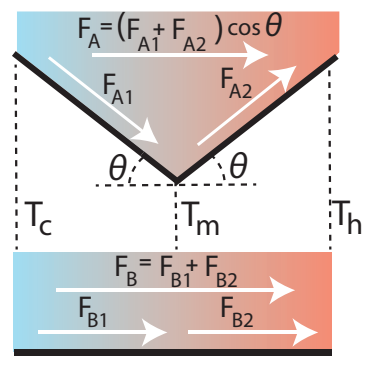

FIG. 9. Sketch illustrating the decrease of the thermophoretic force along an indented surface.

perpendicular to the temperature gradient. We therefore define the dimensionless thermophoretic anisotropy factor as

$$
\chi_{T}=\alpha_{T, \perp}-\alpha_{T, \|} \cdot
$$

The intensity and the sign of the force perpendicular to the temperature gradient is simply determined by $\chi_{T}$, as already shown in Eq. (6). The direction of the perpendicular force will have crucial importance in applications of the effect, determining for example the rotation direction of the thermophoretic turbines [14. This means that the sign of $\alpha_{T}^{0}$ will not be enough to know the direction of the perpendicular force; or, in other words, the thermophilic or thermophobic character of the surface does not determine the direction of the transverse phoretic effect.

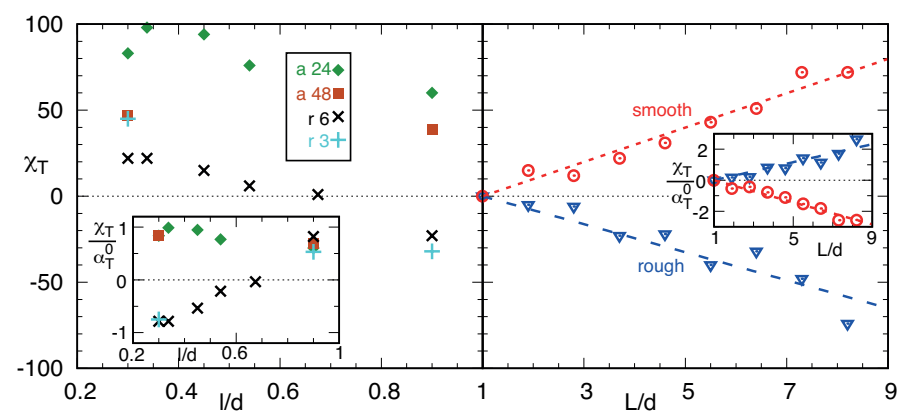

FIG. 10. a) Asymmetric thermophoretic factor $\chi_{T}$ as function of the rod rugosity parameter, for various interaction potentials, with $L / d=3.7$. b) $\chi_{T}$ as a function of the aspect ratio for $r 6$ interaction potential and rugosities $L / d=0.3$ and 0.9 . The insets correspond to the ratio $\chi_{T} / \alpha_{T}^{0}$ for the same data.

The value of $\chi_{T}$ is displayed for various potentials and rugosities in Fig. 10, where it can be observed that $\chi_{T}>0$ in the majority of the cases. This means that the perpendicular force happens most of the time in the same direction, which is positive with the convection in Fig. 2. In the thermophobic case, where the thermodiffusion factor is positive, $\chi_{T}>0$ corresponds to $\left|\alpha_{T, \perp}\right|>\left|\alpha_{T, \|}\right|$ what resembles the well-know translational friction case. However, in the thermophilic case, where the thermodiffusion factors are negative $\chi_{T}>0$ 
corresponds to $\left|\alpha_{T, \perp}\right|<\left|\alpha_{T, \|}\right|$. For comparison, the insets in Fig. 10 show the ratio $\chi_{T} / \alpha_{T}^{0}$, which will be positive in the friction-like cases, and negative otherwise. This normalization also provides a rapid estimation of the magnitude of the effect which can be as expected to be as large as the phoretic effect itself, even for the small aspect ratios here considered. Note though that these are the results obtained by means of computer simulation and that in practical experiments, the results can show a much richer behavior. With our simulation results, we observe that by changing the surface rugosity, the anisotropic effect can reverse its direction in the case of thermophilic rods. We expect this behavior to be reproducible experimentally by changing the surface coating, electrostatic interactions, average temperature, or any of the factors that are known to affect the thermophoretic behavior.

\section{CONCLUSIONS}

Anisotropic thermophoresis refers to the different phoretic thrust that an elongated particle suffers when aligned with the temperature gradient and when perpendicular to it. This difference results in a contribution to the thermophoretic force perpendicular to the temperature gradient when the rod is fixed oblique to the gradient. This anisotropy does not have any relevant effect on the particle orientation, nor on the rotational diffusion of the particle, given the considered symmetry of the colloidal rod. Although unreported until know, the existence of anisotropic thermophoresis is relatively intuitive, especially by comparison with the translational friction of a rod which is also noticeable different if aligned with the flow, or perpendicular to it. Here we analyze this effect in detail, showing that the intensity and the direction of the force is a function of the aspect ratio, the surface geometry, and the colloid-fluid interactions. Increasing aspect ratio linearly increases the anisotropic phoretic effect in an straightforward manner. The rugosity of the colloidal surface is also relevant, being smooth surfaces the ones with larger anisotropic thermophoretic effect. In general, surfaces with larger phoretic effect also have larger anisotropic phoretic effect. Interestingly, and for the simulation potentials employed in this work, the direction of the phoretic force perpendicular to the gradient is the same for both thermophobic and thermophilic colloids. Only rods with thermophilic and rough surface have shown in our simulations to display the perpendicular forces with opposite direction. We expect that experimental results will show an even richer behavior, in which the intensity and the direction of the effect could depend on many factors such as intrinsic surface properties, eventual coatings, average fluid temperature and density, presence of salt ions, and various other factors. Two first practical applications of anisotropic thermophoresis have already been found in the construction of phoretic microturbines and micropumps. In the presence of external temperature gradients, the blades of a microturbine will rotate when being anisotropic [14, and a microchannel will experience some spontaneous directed fluid motion when including elongated tilted obstacles [57]. Although the work presented here has been exclusively focused in the thermophoretic effect, very similar results are expected with other phoretic effect such as diffusiophoresis. A direct proof for this is the fact that a similar micro-turbine placed in an external concentration gradient has shown to display similar behavior due to the related anisotropic diffusiophoresis [58. In summary, with this investigation, we provide a deep insight into the anisotropic thermophoresis of elongated micro-meter size objects; effect that we hope will be soon experimentally verified, and find applications in different fields like particle characterization, microfluidics, or biomedicine.

\section{ACKNOWLEDGMENTS}

We gratefully thank the computing time granted on the supercomputer JURECA at Jülich Supercomputing Centre (JSC). Financial support from China Scholarship Council (CSC) is gratefully acknowledged. M. Y. also acknowledges financial support of the NSFC (No. 11674365).
[1] C. Ludwig, Sitzungsber. Preuss. Akad. Wiss., Phys. Math. Kl. 20, 539 (1856).

[2] C. Soret, Arch. Sci. Phys. Nat. 3, 48 (1879).

[3] S. Wiegand, J. Phys.: Condens. Matter 16, R357 (2004).

[4] R. Piazza and A. Parola, J. Phys.: Condens. Matter 20, 153102 (2008).

[5] A. Würger, Rep. Prog. Phys. 73, 126601 (2010).

[6] D. Reith and F. Müller-Plathe, J. Chem. Phys. 112, 2436 (2000).

[7] C. Debuschewitz and W. Köhler, Phys. Rev. Lett. 87, 055901 (2001).

[8] G. Galliéro, B. Duguay, J.-P. Caltagirone, and F. Montel, Fluid Phase Equilibria 208, 171 (2003).
[9] P. Blanco, H. Kriegs, M. P. Lettinga, P. Holmqvist, and S. Wiegand, Biomacromolecules 12, 1602 (2011).

[10] Z. Wang, H. Kriegs, J. B. J. K. G. Dhont, and S. Wiegand, Soft Matter 9, 8697 (2013).

[11] S. Duhr and D. Braun, Phys. Rev. Lett. 96, 168301 (2006).

[12] S. Iacopini, R. Rusconi, and R. Piazza, Eur. Phys. J. E 19, 59 (2006).

[13] M. Doi and S. F. Edwards, The Theory of Polymer Dynamics (Oxford University Press, Oxford, 1986).

[14] M. Yang, R. Liu, M. Ripoll, and K. Chen, Nanoscale 6, 13550 (2014). 
[15] K. Ghorayeb, A. Firoozabadi, and T. Anraku, SPE Journal 18, 114 (2003).

[16] J. Giddings, Science 260, 1456 (1993).

[17] P. Baaske, F. M. Weinert, S. Duhr, K. H. Lemke, M. J. Russell, and D. Braun, Proc. Natl. Acad. Sci. 104, 9346 (2007).

[18] D. Niether, D. Afanasenkau, J. K. G. Dhont, and S. Wiegand, Proc. Natl. Acad. Sci. 113, 4272 (2016).

[19] M. Jerabek-Willemsen, T. André, R. Wanner, H. M. Roth, S. Duhr, P. Baaske, and D. Breitsprecher, J. Mol. Struc. 1077, 101 (2014).

[20] D. Vigolo, R. Rusconi, H. A. Stone, and R. Piazza, Soft Matter 6, 3489 (2010).

[21] M. Yang and M. Ripoll, Soft Matter 12, 8564 (2016).

[22] H. R. Jiang, N. Yoshinaga, and M. Sano, Phys. Rev. Lett. 105, 268302 (2010).

[23] M. Yang and M. Ripoll, Phys. Rev. E 84, 061401 (2011).

[24] M. Yang, A. Wysocki, and M. Ripoll, Soft Matter 10, 6208 (2014).

[25] A. Malevanets and R. Kapral, J. Chem. Phys. 110, 8605 (1999).

[26] A. Malevanets and R. Kapral, J. Chem. Phys. 112, 7260 (2000).

[27] R. Kapral, Adv. Chem. Phys. 140, 89 (2008).

[28] T. Ihle and D. M. Kroll, Phys. Rev. E 63, 020201(R) (2001).

[29] M. Ripoll, K. Mussawisade, R. G. Winkler, and G. Gompper, Phys. Rev. E 72, 016701 (2005).

[30] E. Tüzel, T. Ihle, and D. M. Kroll, Phys. Rev. E 74, 056702 (2006).

[31] I. O. Götze, H. Noguchi, and G. Gompper, Phys. Rev. E 76, 046705 (2007).

[32] H. Noguchi, N. Kikuchi, and G. Gompper, Europhys. Lett. 78, 10005 (2007).

[33] M. Yang, M. Theers, J. Hu, G. Gompper, R. G. Winkler, and M. Ripoll, Phys. Rev. E 92, 013301 (2015).

[34] N. Kikuchi, C. M. Pooley, J. F. Ryder, and J. M. Yeomans, J. Chem. Phys. 119, 6388 (2003).

[35] C. M. Pooley and J. M. Yeomans, J.Phys. Chem.B 109, 6505 (2005).
[36] T. Ihle, E. Tüzel, and D. M. Kroll, Phys. Rev. E 72, 046707 (2005).

[37] H. Noguchi, N. Kikuchi, and G. Gompper, Europhys. Lett. 79, 36002 (2007).

[38] R. G. Winkler and C.-C. Huang, J. Chem. Phys. 130, 074907 (2009).

[39] C. Huang, A. Varghese, G. Gompper, and R. Winkler, Phys. Rev. E 91, 013310 (2015).

[40] D. Lüsebrink and M. Ripoll, J. Chem. Phys. 136, 084106 (2012).

[41] M. Yang and M. Ripoll, Soft Matter 9, 4661 (2013).

[42] F. Müller-Plathe, J. Chem. Phys. 106, 6082 (1997).

[43] G. A. Vliegenthart, J. F. M. Lodge, and H. N. W. Lekkerkerker, Physica A 263, 378 (1999).

[44] D. Lüsebrink, M. Yang, and M. Ripoll, J. Phys.: Condens. Matter 24, 284132 (2012).

[45] M. P. Allen and D. J. Tildesley, Computer Simulations in Liquids (Clarendon, Oxford, 1987).

[46] A. Parola and R. Piazza, J. Phys.: Condens. Matter 17, S3639 (2005).

[47] M. Yang and M. Ripoll, J. Phys.: Condens. Matter 24, 195101 (2012).

[48] M. Yang and M. Ripoll, J. Chem. Phys. 136, 204508 (2012).

[49] H. R. Jiang, H. Wada, N. Yoshinaga, and M. Sano, Phys. Rev. Lett. 102, 208301 (2009).

[50] L. Helden, R. Eichhorn, and C. Bechinger, Soft Matter 11, 2379 (2015).

[51] T. Ihle and D. M. Kroll, Phys. Rev. E 67, 066706 (2003).

[52] F. Römer, F. Bresme, J. Muscatello, D. Bedeaux, and J. M. Rubí, Phys. Rev. Lett. 108, 105901 (2012).

[53] C. D. Daub, P.-O. ØAstranda, and F. Bresme, Phys. Chem. Chem. Phys. 16, 22097 (2014).

[54] A. A. Lee, Soft Matter 12, 8661 (2016).

[55] M. Braibanti, D. Vigolo, and R. Piazza, Phys. Rev. Lett. 100, 108303 (2008).

[56] S. Duhr and D. Braun, Proc. Natl. Acad. Sci. 103, 19678 (2006).

[57] Z. Tan, M. Yang, and M. Ripoll, (preprint, 2017).

[58] M. Yang, R. Liu, M. Ripoll, and K. Chen, Lab Chip 15, 3912 (2015). 